\title{
Frustration and Entanglement in Compass and Spin-Orbital Models
}

\author{
ANDRZEJ M. Oleś* \\ M. Smoluchowski Institute of Physics, Jagiellonian University, prof. S. Łojasiewicza 11, PL-30-348 Kraków, Poland \\ and \\ Max-Planck-Institut für Festkörperforschung, Heisenbergstraße 1, D-70569 Stuttgart, Germany
}

\begin{abstract}
We review the consequences of intrinsic frustration of the orbital superexchange and of spin-orbital entanglement. While the Heisenberg perturbing interactions remove frustration in the compass model, the lowest columnar excitations are robust in the nanoscopic compass clusters and might be used for quantum computations. Entangled spin-orbital states determine the ground states in some cases, while in others concern excited states and lead to measurable consequences, as in the $\mathrm{RVO}_{3}$ perovskites. On-site entanglement for strong spin-orbit coupling generates the frustrated Kitaev-Heisenberg model with a rich magnetic phase diagram on the honeycomb lattice. Frustration is here reflected in hole propagation which changes from coherent in an antiferromagnet via hidden quasiparticles in zigzag and stripe phases to entirely incoherent one in the Kitaev spin liquid.
\end{abstract}

DOI: 10.12693/APhysPolA.127.163

PACS: 75.10.Jm, 64.70.Tg, 75.25.Dk, 79.60.-i

\section{Introduction}

Over the last decade the spin-orbital physics developed to a very active and challenging field which unifies frustrated magnetism and the phenomena in strongly correlated electron systems. It arose from the pioneering ideas of Kugel and Khomskii who recognized that spins and orbitals have to be treated on equal footing as quantum operators in transition metal oxides with partly filled degenerate $3 d$ orbitals at large Coulomb interaction $U$ [1]. In Mott and charge-transfer insulators, the a priori coupled spin-orbital degrees of freedom interact on the three-dimensional (3D) cubic lattice via the superexchange which follows from degenerate Hubbard model [2] and takes the form of a generalized Heisenberg model [3],

$$
\mathcal{H}=\sum_{\langle i j\rangle \| \gamma}\left\{J_{i j}^{(\gamma)}\left(\boldsymbol{\tau}_{i}, \boldsymbol{\tau}_{j}\right) \boldsymbol{S}_{i} \cdot \boldsymbol{S}_{j}+K_{i j}^{(\gamma)}\left(\boldsymbol{\tau}_{i}, \boldsymbol{\tau}_{j}\right)\right\} .
$$

Here the operators $J_{i j}^{(\gamma)}$ and $K_{i j}^{(\gamma)}$ determine the Heisenberg exchange between spins $\boldsymbol{S}_{i} \equiv\left\{S_{i}^{x}, S_{i}^{y}, S_{i}^{z}\right\}$ - they depend on the bond direction $\gamma=a, b, c$ in the cubic lattice via the orbital operators $\left\{\boldsymbol{\tau}_{i}, \boldsymbol{\tau}_{j}\right\}$ at sites $i$ and $j$. Spin-orbital models (SOMs) relevant for real materials are quite involved and depend on whether the orbital degrees of freedom are $e_{g}$ or $t_{2 g}$. They follow from virtual charge excitations along the bonds $\langle i j\rangle[3]$ and include the multiplet structure of excited states. Quantum fluctuations are of particular importance in $t_{2 g}$ systems where two orbitals are active along each bond $[4,5]$. In the case of large spins in the colossal magnetoresistance manganites with $S=2$ spins [6], spins and orbitals nearly

\footnotetext{
*e-mail: a.m.oles@uj.edu.pl
}

decouple and the $A$-type antiferromagnetic (AF) and ferromagnetic (FM) phase are well understood [7]. In spite of this decoupling of spins from orbitals, several questions remain, as for instance the theoretical explanation of the phase diagram of insulating manganites [8]. Even more challenging are systems with small spins, with their properties determined by spin-orbital entanglement (SOE) [9].

While the intrinsic frustration of orbital interactions may be released by emerging spin-orbital order, the difference between spins and orbitals is best understood by considering generic orbital models, as the twodimensional (2D) compass model [10] and the Kitaev model on the honeycomb lattice [11]. Both may be derived as limiting cases of magnetic interactions in the Mott-Hubbard systems with partially filled $t_{2 g}$ levels and with strong spin-orbit coupling [12] — then SOE occurs on-site and leads to a rich variety of the low energy Hamiltonians that extrapolate from the Heisenberg to a quantum compass or Kitaev model. Yet, these two models are quite different - the $2 \mathrm{D}$ compass model has one-dimensional (1D) nematic order at finite temperature [13], while the exact solution of the Kitaev model is instead a disordered Kitaev spin liquid (KSL) with only nearest neighbor (NN) spin correlations. Realistic 2D or $3 \mathrm{D} e_{g}$ orbital models are also strongly frustrated, but orbitals order at finite temperature following the strongest interactions [14], while quantum effects are small.

The purpose of this paper is to summarize selected recent developments presented at PM'14 Conference. We discuss the phase diagram of the compass-Heisenberg (CH) model in Sect. 2. Next we present a few examples of SOE in $1 \mathrm{D}$ and $2 \mathrm{D}$ systems, and in the $\mathrm{RVO}_{3}$ perovskites (where $\mathrm{R}=\mathrm{Lu}, \mathrm{Yb}, \ldots, \mathrm{La}$ ) in Sect. 3. The case of strong spin-orbit coupling realized in $\mathrm{Na}_{2} \mathrm{IrO}_{3}$ and frustrated interactions on the honeycomb lattice are analyzed in Sect. 4. The paper is summarized in Sect. 5 . 


\section{Frustration in compass models}

Although the 2D Ising and compass model are in the same universality class, they are quite different - the first one is classical, while in the second one two pseudospin components $\left\{\tau_{i}^{x}, \tau_{i}^{z}\right\}$ interact either along horizontal or along vertical bonds by $J_{x}$ and $J_{z}$, and the ground state is highly degenerate and has $1 \mathrm{D}$ columnar order. Evolution between these two limits was investigated by the multiscale entanglement renormalization Ansatz and a quantum phase transition (QPT) from the 2D FM (AF) to nematic order was found close to the compass limit [15].

Another QPT occurs in the compass model itself for increasing $\left|J_{x}\right| / J_{z}$ at $\left|J_{x}\right|=J_{z}$, when the $1 \mathrm{D}$ order switches from vertical to horizontal bonds [16]. Understanding of symmetries in the $2 \mathrm{D}$ compass model allows one to calculate exact spectra of $L \times L$ clusters (with $L=6$ ) by mapping them to $(L-1) \times(L-1)$ clusters with modified interactions and to uncover the hidden dimer order [17].

The nematic order and the above hidden order in the $2 \mathrm{D}$ compass model are fragile and disappear in presence of infinitesimally small Heisenberg interaction $\propto I[18]$. The $\mathrm{CH}$ model (we take $J_{z}>0$ ),

$$
\begin{aligned}
& H_{\mathrm{CH}}=J_{x} \sum_{i, j} \tau_{i, j}^{z} \tau_{i+1, j}^{z}+J_{z} \sum_{i, j} \tau_{i, j}^{x} \tau_{i, j+1}^{x} \\
& \quad+I \sum_{i, j} \boldsymbol{\tau}_{i, j} \cdot\left(\boldsymbol{\tau}_{i, j+1}+\boldsymbol{\tau}_{i+1, j}\right),
\end{aligned}
$$

has a very rich phase diagram (Fig. 1) and the symmetry breaking involves the component $\tau_{i, j}^{\alpha}(\alpha=x, z)$ with the strongest interactions. As both AF and FM interactions are possible, one finds also $C$-type $\mathrm{AF}(C$-AF $)$ order, with AF order between FM lines. The QPTs follow mostly from symmetry and are thus given by straight lines. Surprisingly, however, the nematic order survives

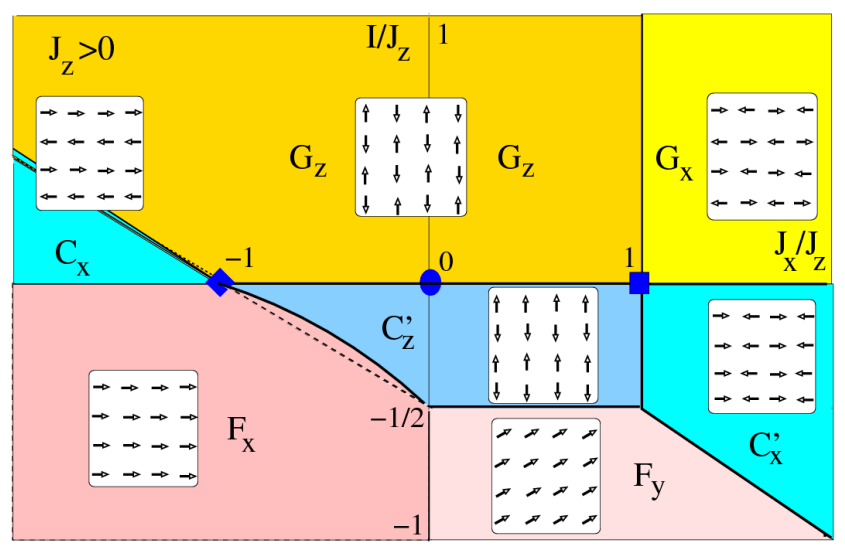

Fig. 1. Phase diagram of the $\mathrm{CH}$ model in the $\left(J_{x}, I\right)$ plane for fixed AF interaction $J_{z}=1$. Long-range spin order in phases $\left\{G_{z}, G_{x}, C_{z}^{\prime}, C_{x}, C_{x}^{\prime}, F_{x}, F_{y}\right\}$ (the subscript $\alpha=x, y, z$ indicates the order parameter), depicted in a corresponding inset, replaces the nematic order for any finite $I$. Square $\left(J_{x}=J_{z}\right)$ and diamond $\left(J_{x}=-J_{z}\right)$ at the compass line $(I=0)$ indicate multicritical points. The quantum corrections contribute to the QPTs between $F_{x}$ and $C_{z}^{\prime}\left(C_{x}\right.$ and $\left.G_{z}\right)$ phases (solid lines). This figure is reproduced from [18]. in the excited states in finite clusters, with somewhat lower quantum fluctuations for FM couplings $J_{\alpha}<0$. Indeed, this case should be of more importance for possible applications in quantum computing as information is easy to store by applying magnetic field when nematic order is FM. Crucial for these applications is large gap in spin excitations which occurs in the anisotropic $X Y Z$ Heisenberg model (2). Therefore the columnar compass excited states are the lowest energy excitations in a broad range of parameters, when the perturbation $\propto I$ is weak and the cluster size is nanoscopic [18]. Certain realizations of computing devices with protected qubits have been implemented in the Josephson junction arrays [19], while systems of trapped ions in optical lattices look also promising [20].

In the $1 \mathrm{D}$ compass model the consequences of frustration can be studied exactly, and one finds a QPT between two types of order on even/odd bonds at the $J_{x}=J_{z}$ point [21]. This model is quite distinct from the orbital $e_{g}$ model for a zigzag (ZZ) chain where frustration is weaker - recent studies uncover rather peculiar behavior in the thermodynamic properties of the $1 \mathrm{D}$ compass model which follow from highly frustrated interactions [22]. An exact solution is also possible for a compass ladder [23], which elucidates the nature of the QPT from ordered to disordered ground state found in the $2 \mathrm{D}$ compass model. Another type of frustration is encountered in the 1D plaquette compass model, where exact solution is no longer possible due to entanglement which increases locally in excited states and coincides with disorder [24].

The $2 \mathrm{D}$ compass model can be seen as the strongcoupling limit of a spinless two-band Hubbard model with nonequivalent hopping matrices for the bonds along the $a$ and $b$ axis in the square lattice. Therefore, a hole is not confined in the nematic state of the $2 \mathrm{D}$ compass model [25], unlike in the $2 \mathrm{D}$ Ising limit or in the $2 \mathrm{D} t_{2 g}$ orbital model [26]. The qualitative change of the hole excitation spectra near the nematic state corresponds to the QPT. An important common feature of the 2D orbital and compass model is that quantum fluctuations are absent, and therefore the kinetic energy plays a particularly important role. It reorients the orbitals in the $2 \mathrm{D}$ alternating orbital ( $\mathrm{AO}$ ) state into ferro-orbital (FO) ordered domain walls that allow for deconfined motion of holes [27], similar to FO order induced locally in a $1 \mathrm{D}$ doped $e_{g}$ system (manganite) [28].

\section{Entanglement in spin-orbital models}

Unless spins are FM, one has to consider orbitals coupled to spins in the framework of general SOMs. In some cases the spin-orbital order is determined by the Kanamori-Goodenough rules stating the spin and orbital order are complementary, but in general SOE is expected. One of the main difficulties is a reliable approach to entangled ground states, as one can see on the example of frustrated exchange on the triangular lattice, where superexchange competes with direct exchange [29]. In the 
disordered ground state with dimer orbital correlations SOE prevents any reliable predictions concerning the magnetic interactions on superexchange bonds, and spin correlations do not follow the sign of the spin exchange obtained using the mean-field (MF) approach [30].

The SOE was discovered in $1 \mathrm{D} d^{1}$ and $d^{2}$ systems with $t_{2 g}$ orbitals [31], but occurs also in $e_{g}$ systems, see below. The Bethe-Ansatz solution of the SU(4) 1D model [32] demonstrates that its ground state and excitations are controlled by SOE. Recently another 1D model has been solved exactly providing a beautiful example of SOE, the $\mathrm{SU}(2) \otimes X Y$ ring [33],

$$
\mathcal{H}_{\mathrm{SU}(2) \otimes X Y}=\frac{1}{2} J \sum_{i=1}^{L}\left(\boldsymbol{\sigma}_{i} \cdot \boldsymbol{\sigma}_{i+1}+1\right)\left(\tau_{i+1}^{+} \tau_{i}^{-}+\tau_{i+1}^{-} \tau_{i}^{+}\right),
$$

where $\sigma_{l}$ 's are spin Pauli matrices, and $\tau_{l}$ 's are orbital Pauli matrices, and $L+1 \equiv 1$. The spin transposition operator, $X_{i, i+1} \equiv\left(\boldsymbol{\sigma}_{i} \cdot \boldsymbol{\sigma}_{i+1}+1\right) / 2$, interchanges spins on the bond $\langle i, i+1\rangle$, i.e., $X_{i, i+1} \boldsymbol{\sigma}_{i} X_{i, i+1}=\boldsymbol{\sigma}_{i+1}$. For an open chain the spins and orbitals are decoupled by a unitary transformation $\mathcal{U}[34]$, spins are disordered and the ground state has a large degeneracy $\mathcal{D}=2^{L}$. Closing the spin-orbital chain to a ring (3) causes surprising changes in the spin part of the lowest-lying eigenstates. All the eigenstates are grouped in multiplets labeled by quasimomenta $\mathcal{K}$, and the ground state has $\mathcal{K}=0$ (Fig. 2). Therefore the topological order emerges and the ground state degeneracy drops to $\mathcal{D}=2^{L+1} / L[33]$.

When the orbital interactions have $\mathrm{SU}(2)$ symmetry,

$$
\mathcal{H}_{\mathrm{SU}(2) \otimes \mathrm{SU}(2)}=\frac{1}{2} J \sum_{\langle i j\rangle}\left(\boldsymbol{S}_{i} \cdot \boldsymbol{S}_{i+1}+x\right)\left(\boldsymbol{\tau}_{i} \cdot \boldsymbol{\tau}_{i+1}+y\right),
$$

one considers instead a 1D SOM (4) with a higher SU(4) symmetry at $x=y=1 / 4$. Recently its phase diagram was investigated numerically for $J<0$ [35]. One finds four phases, with: $\mathrm{FM} / \mathrm{FO}, \mathrm{AF} / \mathrm{FO}, \mathrm{AF} / \mathrm{AO}$, and $\mathrm{FM} / \mathrm{AO}$ order. The $\mathrm{FM} / \mathrm{FO}$ ground state is disentangled, but SOE occurs in excited states. Spin and

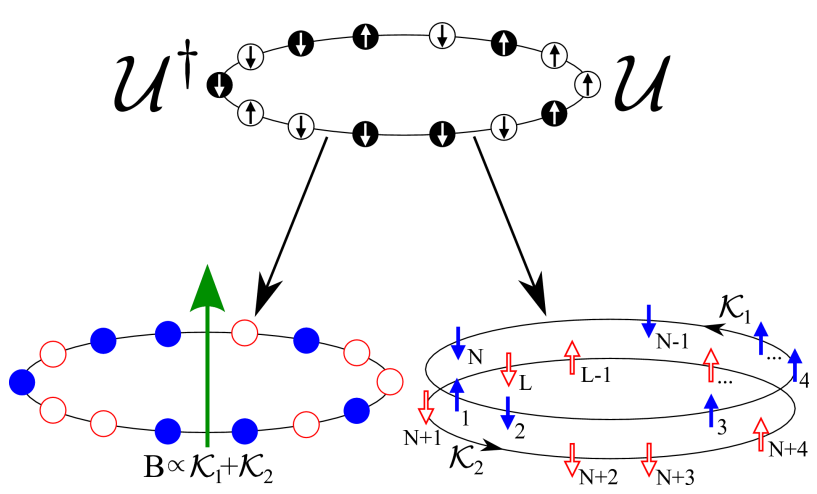

Fig. 2. Artist's view of the spin-orbital decoupling in the ring $(3)$ caused by the transformation $\mathcal{U}$. The initial spin-orbital chain (top) splits into purely orbital (left) and spin (right) segments. The spin part consists of two halves carrying quasimomenta $\mathcal{K}_{1}$ and $\mathcal{K}_{2}$. The orbital part feels an external magnetic field $\boldsymbol{B}$ perpendicular to the ring (arrow). This figure is reproduced from [33]. orbital excitations are entangled in the continuum, as well as a spin-orbital quasiparticle (QP) and bound state. A useful tool to investigate SOE in all these states is von Neumann entropy spectral function which gives the highest entanglement for the latter composite spin-orbital excitations, the QP and the bound state [35]. The scaling of the von Neumann entropy with system size is logarithmic and qualitatively different from other spin-orbital excitations from the continuum, where the entropy saturates.

Another example of SOE is found in the KugelKhomskii (KK) SOM, where exotic types of magnetic order occur [36, 37]. The 2D KK model describes the superexchange $\propto J=4 t^{2} / U$ in $\mathrm{K}_{2} \mathrm{CuF}_{4}$ between holes with $S=1 / 2$ spins in $e_{g}$ orbitals $(\tau=1 / 2)$,

$$
\begin{gathered}
\mathcal{H}_{\mathrm{KK}}=\frac{1}{2} J \sum_{\langle i j\rangle \| \gamma=a b}\left\{-r_{1}\left(\boldsymbol{S}_{i} \cdot \boldsymbol{S}_{j}+\frac{3}{4}\right)\left(\frac{1}{4}-\tau_{i}^{\gamma} \tau_{j}^{\gamma}\right)\right. \\
+r_{2}\left(\boldsymbol{S}_{i} \cdot \boldsymbol{S}_{j}-\frac{1}{4}\right)\left(\frac{1}{4}-\tau_{i}^{\gamma} \tau_{j}^{\gamma}\right) \\
\left.+\left(r_{2}+r_{4}\right)\left(\boldsymbol{S}_{i} \cdot \boldsymbol{S}_{j}-\frac{1}{4}\right)\left(\frac{1}{2}-\tau_{i}^{\gamma}\right)\left(\frac{1}{2}-\tau_{j}^{\gamma}\right)\right\} .
\end{gathered}
$$

where $\tau_{i}^{c}=\tau_{i}^{z}=\sigma_{i}^{z} / 2, \tau_{i}^{a, b}=\left(-\tau_{i}^{z} \pm \sqrt{3} \tau_{i}^{x}\right) / 4$, while $r_{1}=1 /(1-3 \eta), r_{2}=1 /(1-\eta)$, and $r_{4}=1 /(1+\eta)$ follow from the multiplet structure and depend on Hund's exchange $\eta \equiv J_{H} / U$. The second parameter extending the model (5) is the orbital splitting, $H_{z}=E_{z} \sum_{i} \tau_{i}^{z}$.

The phase diagram of the $2 \mathrm{D}$ KK model (5) obtained by two variational methods, a cluster MF (CMF) and entanglement renormalization Ansatz (ERA), contains the exotic magnetic order (ortho-AF phase in Fig. 3) between the AF and FM phase for $E_{z}<0$ and

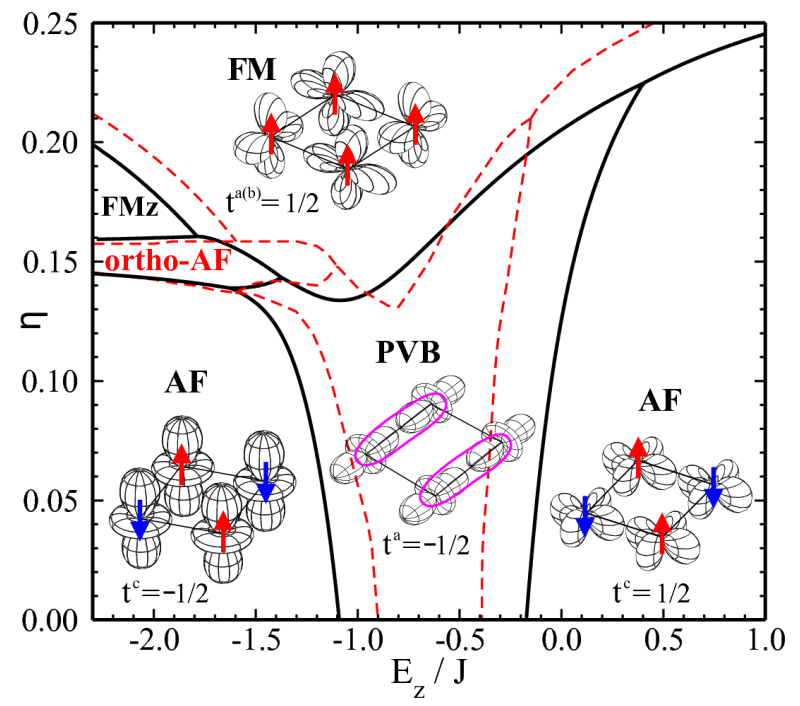

Fig. 3. Phase diagram of the 2D KK model in the $\mathrm{CMF}$ and ERA (solid and dashed lines). Insets indicate spin-orbital configurations on a $2 \times 2$ plaquette $x$-like $\left(\tau_{i}^{c}=-1 / 2\right)$ and $z$-like $\left(\tau_{i}^{c, a}=1 / 2\right)$ orbitals are accompanied either by AF spin order (arrows) or by spin singlets (in the PVB phase). The FM phase has either a two-sublattice $\mathrm{AO}$ order or $\mathrm{FO} z$ order $(\mathrm{FM} z)$. An exotic ortho-AF phase separates the AF and FM phases. This figure is reproduced from [36]. 
$\eta \approx 0.155$ [36], where the NN spin exchange changes sign. As shown in the perturbation theory which starts with $3 z^{2}-r^{2}$ orbitals occupied by holes in the ground state and treats $\mathcal{H}_{\mathrm{KK}}$ as perturbation, the next nearest neighbor (NNN) and third nearest neighbor (3NN) spin exchange is necessary to understand the origin of the four-sublattice AF phase (Fig. 3). This ground state is stabilized by local entangled spin-orbital excitations to spin singlets and $x^{2}-y^{2}$ orbitals [36]. Exotic magnetic order is also found in a bilayer and in the $3 \mathrm{D} \mathrm{KK}$ model - it follows again from SOE [37].

In the $\mathrm{RVO}_{3}$ perovskites SOE in excited states decides about the properties observed at finite temperature. While the spins and orbitals and their energy scales are well separated in the $\mathrm{RMnO}_{3}$ perovskites [8], the structural (orbital) and magnetic transition are here at rather similar temperature in $\mathrm{RVO}_{3}$ [38]. The orbital orbital order (OO) transition temperature $T_{\mathrm{OO}}=143 \mathrm{~K}$ is almost the same as the Néel temperature $T_{\mathrm{N} 1}=141 \mathrm{~K}$ in $\mathrm{LaVO}_{3}$, and increases with decreasing ionic radius $r_{R}$. Next it saturates and decreases from $\mathrm{YVO}_{3}$ to $\mathrm{LuVO}_{3}$ whereas $T_{\mathrm{N} 1}$ decreases monotonically along the $\mathrm{RVO}_{3}$ series. A theoretical explanation of these phase transitions requires the full superexchange model given in [39], supplemented by the orbital-lattice interactions [40],

$$
\mathcal{H}_{\text {orb }}=E_{z} \sum_{i} \mathrm{e}^{\mathrm{i} \boldsymbol{R}_{i} \boldsymbol{Q}} \tau_{i}^{z}+V_{a b} \sum_{\langle i j\rangle \| a b} \tau_{i}^{z} \tau_{j}^{z}+g_{\text {eff }} \sum_{i} \tau_{i}^{x}
$$

The leading term in the superexchange along the $c$ axis is similar to Eq. (4), with spin $S=1, x=1, y=\frac{1}{4}$, and orbital $\boldsymbol{\tau}_{i} \equiv\left\{\tau_{i}^{x}, \tau_{i}^{y}, \tau_{i}^{z}\right\}$ operators $(\tau=1 / 2)$ for the active $t_{2 g}$ orbitals, $y z$ and $z x$. The SOE occurs only along the bonds $\langle i j\rangle \| c$ as $x y$ orbitals are occupied at each site and thus orbital fluctuations are blocked along the bonds in the $a b$ planes. The crystal-field splitting term $\propto E_{z}$ supports $C$-type $\mathrm{OO}$ and alternates in the $a b$ planes, with $\boldsymbol{Q}=(\pi, \pi, 0)$. Actually, it competes with the superexchange which induces instead the observed $G$ type OO [38]. The Jahn-Teller term $\propto V_{a b}$ supports as well AO order in the $a b$ planes, while along the $c$ axis FO order is favored by a similar interaction [40], neglected for simplicity in Eq. (6).

Due to SOE, which is activated in the excited states at finite temperature, it is crucial to employ a CMF approach, similar to the one used in KK models [37]. In this approach one determines self-consistently the MF order parameters $\left\{\left\langle S_{i}^{z}\right\rangle,\left\langle\tau_{i}^{z}\right\rangle,\left\langle\tau_{i}^{x}\right\rangle,\left\langle S_{i}^{z} \tau_{i}^{z}\right\rangle\right\}$ by coupling a cluster along the $c$ axis to its neighbors via the MF terms adjusted to the $C$-AF $/ G$-AO structure. The orbital fluctuations along the $c$ axis are very important and reduce significantly the orbital order parameter $\left\langle\tau_{i}^{z}\right\rangle$.

The structural transition at $T_{\mathrm{OO}}$ is explained as follows. In $\mathrm{LaVO}_{3}$ the orthorhombic distortion $u \equiv(b-a) / a$ is small, where $a$ and $b$ are the lattice parameters of the Pbnm structure. Then the values of $T_{\mathrm{N} 1}$ and $T_{\mathrm{OO}}$ are used to establish the variation of model parameters with increasing lattice distortion $u$. All the parameters in $\mathcal{H}_{\text {orb }}$ increase with increasing $u$ due to decreasing ionic size in

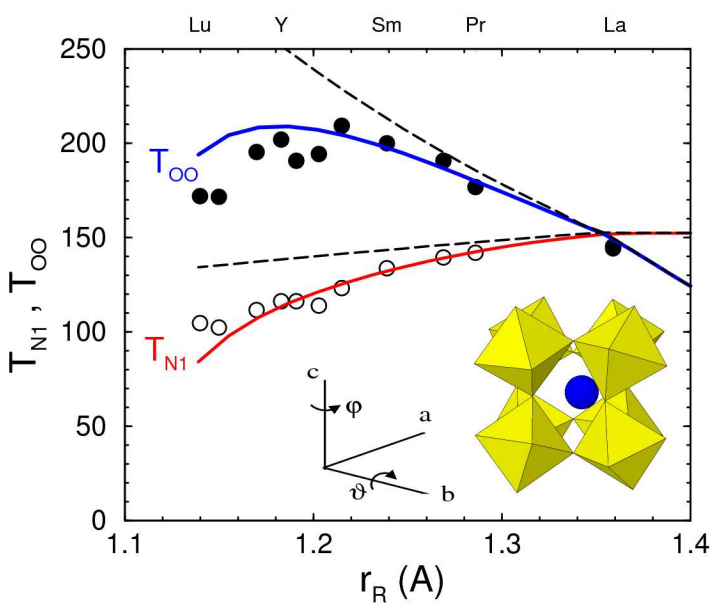

Fig. 4. The orbital $T_{\text {OO }}$ and Néel $T_{N 1}$ transition temperature (solid lines) for varying ionic size in $\mathrm{RVO}_{3}$, as obtained from the theory, and experimental points [38] (full and empty circles). Dashed lines indicate $T_{\mathrm{OO}}$ and $T_{N 1}$ obtained under neglect of orbital-lattice coupling $\left(g_{\text {eff }}=0\right)$. The inset shows the $\mathrm{GdFeO}_{3}$-type distortion, with the rotation angles $\vartheta$ and $\varphi$ as in $\mathrm{YVO}_{3}$. This figure is reproduced from [40].

$\mathrm{RVO}_{3}$ (Fig. 4). This increase is much faster for $g_{\text {eff }}$ than for $E_{z}$ and $V_{a b}$, so from $\mathrm{LaVO}_{3}$ to $\mathrm{SmVO}_{3}$ the latter two parameters alone determine the increase of $T_{\mathrm{OO}}$. When $g_{\text {eff }}$ becomes larger, however, this term acting as a field on the orbitals suppresses partly orbital order, $\left\langle\tau_{i}^{z}\right\rangle$, and the orbital polarization, $\left\langle\tau_{i}^{x}\right\rangle$, increases following local distortions. This reduces $T_{\mathrm{OO}}$ from $\mathrm{YVO}_{3}$ to $\mathrm{LuVO}_{3}$. At the same time $T_{\mathrm{N} 1}$ decreases due to the changes in the orbital order. This decrease would not occur in the absence of lattice distortion (at $u=0$ implying $g_{\text {eff }}=0$ ) which manifests again strong SOE in this system (Fig. 4). We conclude that the lattice distortion $u$, which increases from La to $\mathrm{Y}$ by one order of magnitude, modifies orbital fluctuations and in this way tunes the onset of both orbital and spin order in the cubic vanadates.

There are more experiments which indicate strong SOE in the vanadium perovskites at finite temperature [9]. Here we mention briefly only the dimerization observed in the magnon spectra of the intermediate temperature $C$-AF phase in $\mathrm{YVO}_{3}$ [41]. Spin exchange interactions dimerize as a consequence of the instability of the 1D orbital chain along the $c$ axis. Of course, this mechanism cannot operate at $T=0$ as then the spins have rigid FM order along the $c$ axis. But thermal fluctuations in the spin system weaken spin correlations and dimerization is the way to lower the free energy. We emphasize that the dimerization occurs here simultaneously in both channels but the dimerization in the FM chain (for spins $S=1$ ) is much stronger than in the AO chain [42].

Summarizing, the SOE in the excited states is visible in the magnetic and optical properties of the vanadium perovskites, and any theoretical treatment has to go beyond a simple picture established by the GoodenoughKanamori rules, and one has to go beyond this paradigm 
also in alkali $\mathrm{RO}_{2}$ hyperoxides (with $\mathrm{R}=\mathrm{K}, \mathrm{Rb}, \mathrm{Cs}$ ) [43] or in finite clusters [44]. In the case of quantum states, these rules have to be generalized as follows: In the wave functions a component with spin-singlet and orbital-triplet coexists with a component with spin-triplet and orbitalsinglet. SOE is also of importance for the pairing mechanism in Fe-pnictides [45]. More examples of SOE are presented in [9].

\section{Strong spin-orbit coupling}

In systems with strong spin-orbit coupling, as in iridates, on-site SOE dominates and entangles locally spins and orbitals. In this case one has to determine first effective spins with eigenstates being linear combinations of spin-orbital components [12]. The interactions at low-energy between such effective $S=1 / 2$ spins are in general quite different from superexchange in spinorbital models. Projecting the microscopic interactions on the Kramers doublets gives strongly frustrated interactions [12]: (i) the 2D compass model on the square lattice, and (ii) the Kitaev model on the honeycomb lattice. Kitaev model is a realization of a spin liquid with only NN spin correlations on a nonfrustrated lattice [11]. Similar to the Kitaev model, the triangular lattice of magnetic ions in an $\mathrm{ABO}_{2}$ structure (as for instance in $\mathrm{LiNiO}_{2}$ [46]) has Ising-like interactions $\propto S_{i}^{\alpha} S_{j}^{\alpha}$ with $\alpha=x, y, z$ for three nonequivalent bond directions in the lattice. In what follows we focus on the magnetic interactions on the honeycomb lattice which attracted a lot of attention recently.

Here we consider the Kitaev-Heisenberg (KH) $t-J$ model $(J>0)$ on the honeycomb lattice with two sublattices $A$ and $B$ [47], realized in $\mathrm{Na}_{2} \mathrm{IrO}_{3}$ [48, 49],

$$
\begin{array}{r}
\mathcal{H}_{t J} \equiv t \sum_{\langle i j\rangle \sigma} c_{i \sigma}^{\dagger} c_{j \sigma}+J_{\mathrm{K}} \sum_{\langle i j\rangle \| \gamma} S_{i}^{\gamma} S_{j}^{\gamma}+J_{1} \sum_{\langle i j\rangle} \boldsymbol{S}_{i} \cdot \boldsymbol{S}_{j} \\
+(1-\alpha)\left\{J_{2} \sum_{\{i j\} \in \mathrm{NNN}} \boldsymbol{S}_{i} \cdot \boldsymbol{S}_{j}+J_{3} \sum_{\{i j\} \in 3 \mathrm{NN}} \boldsymbol{S}_{i} \cdot \boldsymbol{S}_{j}\right\}
\end{array}
$$

with FM Kitaev and AF Heisenberg NN exchange,

$$
J_{\mathrm{K}} \equiv-2 J \alpha, \quad J_{1} \equiv J(1-\alpha) \text {. }
$$

The parameter $0 \leq \alpha \leq 1$ interpolates between the Heisenberg and Kitaev model. The NN Kitaev $\left(J_{\mathrm{K}}\right)$ and Heisenberg $\left(J_{1}\right)$ interactions compete in Eq. (7) and the spin order changes with increasing $\alpha$. The signs of these two competing terms (8) are opposite and both AF/FM and FM/AF Heisenberg/Kitaev were studied [50]. Such spin interactions were proposed to describe the Mottinsulating layered iridates [51] — for $J_{1}>0$ also NNN $\left(J_{2}\right)$ and $3 \mathrm{NN}\left(J_{3}\right)$ Heisenberg terms are necessary as only then the experimentally observed ZZ magnetic order in $\mathrm{Na}_{2} \mathrm{IrO}_{3}$ [48] is reproduced. The term $\propto t$ stands for the kinetic energy of composite fermions with pseudospin flavor $\sigma$ in the restricted space which contains no double occupancies.

Consider first a spin order parameter for a phase $\Phi$,

$$
\left.\mathcal{S}_{\Phi}^{2} \equiv \frac{12}{N^{2}} \sum_{i j} \mathrm{e}^{\mathrm{i} \boldsymbol{k} \cdot\left(\boldsymbol{R}_{i}-\boldsymbol{R}_{j}\right)}\left\langle\left(S_{i A}^{z} \pm S_{i B}^{z}\right)\left(S_{j A}^{z} \pm S_{j B}^{z}\right)\right\rangle\right),
$$

where the average is calculated in the ground state $|\Phi\rangle$.
Investigating $\mathcal{S}_{\Phi}$ allows one to identify the symmetry breaking and long-range spin order studying finite clusters where the symmetry broken states do not occur [52]. In the above definition the signs of the spin components $S_{j B}^{z}$ on sublattice $B$ and the vector $\boldsymbol{k}$ are selected differently, depending on the spin order in the considered magnetic phase $\Phi[53]$. Investigating such spin correlations does not suffice to identify the disordered KSL, with finite NN spin correlations. Here we evaluate instead the Kitaev invariant [11] for a single hexagon $\mathcal{C}_{6}$,

$$
\mathcal{L} \equiv 2^{6}\left\langle\prod_{i \in \mathcal{C}_{6}} S_{i}^{\gamma}\right\rangle
$$

At fixed $J_{3}=0.4 J$ one finds first large AF spin correlations $\mathcal{S}_{\mathrm{AF}}$ for $\alpha<0.5$, and next the $\mathrm{ZZ}$ phase is favored for $\alpha>0.5$, as indicated by large $\mathcal{S}_{\mathrm{AF}}$ or $\mathcal{S}_{\mathrm{ZZ}}$ (Fig. 5a). The AF $\leftrightarrow \mathrm{ZZ}$ transition at $\alpha=0.5$ follows from symmetry and is independent of the cluster size. Both $\mathcal{S}_{\mathrm{AF}}$ and $\mathcal{S}_{\mathrm{ZZ}}$ decrease for $\alpha>0.85$ when the ground state of the KH model (7) approaches the KSL at $\alpha \rightarrow 1$, and $\mathcal{L} \rightarrow 1$.

The phase diagram of the $\mathrm{KH}$ model in the $\left(\alpha, J_{3} / J\right)$ plane was determined [53] by analyzing spin order parameters, $\mathcal{S}_{\Phi}^{2}$, and the fidelity susceptibility. It contains four magnetic phases at $J_{2}=0$, the $\mathrm{AF}, \mathrm{ZZ}$, stripe (ST) phase, and the KSL disordered phase (Fig. 5b). The AF phase is stable for small $\alpha$, while at intermediate $\alpha$ it is replaced by two other magnetic phases, ST and ZZ. These types of order with coexisting AF and FM bonds manifest enhanced frustration for increasing Kitaev interactions. The gapless KSL takes over at $\alpha>0.85$ and is also stable in presence of lattice distortions [54]. As shown in the CMF approach, the AF phase is also destabilized by increasing NNN interactions $J_{2}$ [55]. The phase diagram of the $\mathrm{KH}$ model (7) is investigated by several groups at present; further results are given in [56].

Motivated by strongly incoherent photoemission (PES) spectra found in $\mathrm{Na}_{2} \mathrm{IrO}_{3}$ [49], we have used the Lanczos diagonalization of the $N=24$ site cluster with periodic boundary conditions to study the evolution of hole

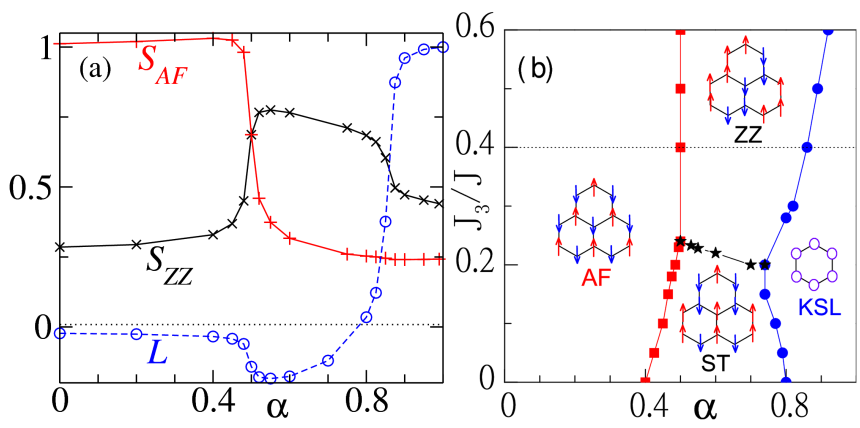

Fig. 5. Magnetic phases in the KH model Eq. (7) shown in: (a) spin correlations $\mathcal{S}_{\Phi}(9)$ representing the $\mathrm{AF}$ and $\mathrm{ZZ}$ order $(\Phi=\mathrm{AF}, \Phi=\mathrm{ZZ})$, and the Kitaev invariant $\mathcal{L}(10)$ for $J_{2}=0, J_{3}=0.4 J$; (b) phase diagram in the $\left(\alpha, J_{3} / J\right)$ plane (points) for $J_{2}=0$, with $\mathrm{AF}$, $\mathrm{ST}, \mathrm{ZZ}$ spin order and KSL disordered phase. The insets show spin order (arrows) or disorder (circles). This figure is reproduced from [53]. 
spectral functions for varying interactions in the $\mathrm{KH}$ model (7) [53]. Indeed, the spectra observed in PES for $\mathrm{Na}_{2} \mathrm{IrO}_{3}$ are rather unexpected as in spite of $\mathrm{ZZ}$ spin order, no QPs and only incoherent spectra are observed. A systematic study of hole spectral properties in this magnetic phase requires to consider two distinct Green's and two spectral functions [57]: (i) full spectral function which corresponds to the PES, and (ii) the sublattice spectral function, i.e., when a hole moves over sites of one sublattice only. One finds that in the $\mathrm{ZZ}$ phase the QPs appear only in the sublattice spectral function while they are hidden in the PES spectral function. This result is independent of the model used to stabilize the $\mathrm{ZZ}$ order and may be considered as following from symmetry of the honeycomb lattice which supports destructive interference in the PES spectral function at low energy [57].

As expected, one finds coherent QPs in the PES spectral function for the AF phase at weakly frustrated interactions, but a similar interference and hidden QPs are found in the ST phase [53]. It is interesting to ask what will happen when spin iteractions are maximally frustrated and the ground state is the KSL. A naive argument that in the absence of robust spin order, quantum spin fluctuations will not couple to the moving hole to generate coherent propagation turns out to be correct, and using exact diagonalization one finds indeed no coherent QPs here, also in the sublattice spectral function. This result was derived by analyzing the sublattice spectral function in absence of spectral broadening [53], $A_{d}(\boldsymbol{k}, \omega)=\sum_{n} \alpha_{d}\left(\boldsymbol{k}, \omega_{n}\right) \delta\left(\omega-\omega_{n}\right)$. The spectral weights $\left\{\alpha_{d}\left(\boldsymbol{k}, \omega_{n}\right)\right\}$ are totally incoherent at low excitation energies $\omega_{n}$, except at the $\boldsymbol{k}=\Gamma$ point in the momentum space [53]. This important result follows from the presence of vortex gap in the Majorana excitations [53] and implies that Ising-like NN spin correlations in the KSL phase are insufficient to generate coherent hole propagation and thus carrier motion in the lightly doped KSL is non-Fermi liquid like. This analysis allows one to conclude [53] that gapless Majorana excitations are responsible for the absence of QPs in the close vicinity of the $\Gamma$ point and, on the contrary to some earlier claims, the weakly doped KSL is not a Fermi liquid.

\section{Summary and outlook}

We have shown that orbital superexchange interactions have lower symmetry than spin ones - they are directional and intrinsically frustrated, also on geometrically nonfrustrated lattices. This leads to nematic order and provides new opportunities for quantum computing. The nematic order in the $2 \mathrm{D}$ compass model is robust and survives in excited states which could be used for storing information in nanoscopic systems, while the Heisenberg perturbing interactions remove frustration and trigger long-range order in the ground state.

In spin-orbital systems frustration in the orbital channel is frequently removed by spin order which modifies the exchange in the orbital subsystem. Nevertheless, $\mathrm{SOE}$ is generic in these systems and may have measurable consequences at finite temperature, as in the $\mathrm{RVO}_{3}$ perovskites. Here, similar to 1D spin-orbital model systems, frustration and entanglement occur simultaneously. In contrast, in systems with strong spin-orbit interaction entanglement comes first and generates frustration as shown on the example of the KH model on the honeycomb lattice. We have also shown that the spectral functions obtained from the $\mathrm{KH}$ model with frustrated interactions describe hidden QPs in the ordered phases with coexisting $\mathrm{FM}$ and $\mathrm{AF}$ bonds, while the Ising-like short-range spin correlations in the KSL are insufficient to generate coherent hole propagation.

We have presented only selected recent developments in the field of spin-orbital physics. Among others, we would like to mention models which describe interfaces or heterostructures, and hybrid bonds between ions with different fillings of the $d$ shell. For instance, $d^{3}$ impurities generate orbital dilution and frustrated interactions in $d^{4}$ systems. Having no orbital degree of freedom, they are able to modify locally orbital order, and the actual spinorbital order in ruthenates might even totally change at finite doping [58]. Summarizing, the spin-orbital physics is a very active and fast developing field of frustrated magnetism with numerous challenging and timely problems, both in the experiment and in the theory. We apologize for not including here many other interesting developments in this field due to the lack of space.

\section{Acknowledgments}

It is our pleasure to acknowledge collaboration and valuable discussions with W. Brzezicki, J. Chaloupka, L. Cincio, M. Cuoco, M. Daghofer, J. Dziarmaga, R. Frésard, A. Herzog, B. Normand, J. Sirker, J. van den Brink, F. Trousselet, K. Wohlfeld, and W.L. You. I thank particularly warmly Louis Felix Feiner, Peter Horsch, and Giniyat Khaliullin for a very friendly and insightful collaboration. We kindly acknowledge financial support by the Polish National Science Center (NCN) under Project No. 2012/04/A/ST3/00331.

\section{References}

[1] K.I. Kugel, D.I. Khomskii, Sov. Phys. Usp. 25, 231 (1982).

[2] A.M. Oleś, Phys. Rev. B 28, 327 (1983).

[3] A.M. Oleś, G. Khaliullin, P. Horsch, L.F. Feiner, Phys. Rev. B 72, 214431 (2005).

[4] G. Khaliullin, Prog. Theor. Phys. Suppl. 160, 155 (2005).

[5] A.M. Oleś, Acta Phys. Pol. A 115, 36 (2009).

[6] E. Dagotto, T. Hotta, A. Moreo, Phys. Rep. 344, 1 (2001); E. Dagotto, New J. Phys. 7, 67 (2005).

[7] L.F. Feiner, A.M. Oleś, Phys. Rev. B 59, 3295 (1999); Phys. Rev. B 71, 144422 (2005); A.M. Oleś, L.F. Feiner, Phys. Rev. B 65, 052414 (2002).

[8] J.-S. Zhou, J.B. Goodenough, Phys. Rev. Lett. 96, 247202 (2006).

[9] A.M. Oleś, J. Phys. Condens. Matter 24, 313201 (2012).

[10] Z. Nussinov, J. van den Brink, Rev. Mod. Phys. 87, 1 (2015).

[11] A.Y. Kitaev, Ann. Phys. (New York) 321, 2 (2006). 
[12] G. Jackeli, G. Khaliullin, Phys. Rev. Lett. 102 , 017205 (2009).

[13] S. Wenzel, W. Janke, Phys. Rev. B 78, 064402 (2008).

[14] A. van Rynbach, S. Todo, S. Trebst, Phys. Rev. Lett. 105, 146402 (2010).

[15] L. Cincio, J. Dziarmaga, A.M. Oleś, Phys. Rev. B 82, 104416 (2010)

[16] R. Orús, A.C. Doherty, G. Vidal, Phys. Rev. Lett. 102, 077203 (2009).

[17] W. Brzezicki, A.M. Oleś, Phys. Rev. B 82, 060401 (2010); Phys. Rev. B 87, 214421 (2013).

[18] F. Trousselet, A.M. Oleś, P. Horsch, Europhys. Lett. 91, 40005 (2010); Phys. Rev. B 86, 134412 (2012).

[19] S. Gladchenko, D. Olaya, E. Dupont-Ferrier, B. Douçot, L.B. Ioffe, M.E. Gershenson, Nature Phys. 5, 48 (2009).

[20] P. Milman, W. Maineult, S. Guibal, L. Guidoni, B. Douçot, L. Ioffe, T. Coudreau, Phys. Rev. Lett. 99, 020503 (2007)

[21] W. Brzezicki, J. Dziarmaga, A.M. Oleś, Phys. Rev. B 75, 134415 (2007); W. Brzezicki, A.M. Oleś, Acta Phys. Pol. A 115, 162 (2009).

[22] W.-L. You, P. Horsch, A.M. Oleś, Phys. Rev. B 89, 104425 (2014); W.-L. You, G.-H. Liu, P. Horsch, A.M. Oleś, Phys. Rev. B 90, 094413 (2014).

[23] W. Brzezicki, A.M. Oleś, Phys. Rev. B 80, 014405 (2009).

[24] W. Brzezicki, A.M. Oleś, Phys. Rev. B 90, 024433 (2014).

[25] W. Brzezicki, M. Daghofer, A.M. Oleś, Phys. Rev. B 89, 024417 (2014); Acta Phys. Pol. A 127, 263 (2015).

[26] M. Daghofer, K. Wohlfeld, A.M. Oleś, E. Arrigoni, P. Horsch, Phys. Rev. Lett. 100, 066403 (2008); K. Wohlfeld, M. Daghofer, A.M. Oleś, P. Horsch, Phys. Rev. B 78, 214423 (2008).

[27] P. Wróbel, A.M. Oleś, Phys. Rev. Lett. 104, 206401 (2010).

[28] M. Daghofer, A.M. Oleś, W. von der Linden, Phys. Rev. B 70, 184430 (2004); M. Daghofer, A.M. Oleś, Acta Phys. Pol. A 111, 497 (2007).

[29] B. Normand, A.M. Oleś, Phys. Rev. B 78, 094427 (2008); B. Normand, Phys. Rev. B 83, 064413 (2011).

[30] J. Chaloupka, A.M. Oleś, Phys. Rev. B 83, 094406 (2011); A.M. Oleś, J. Chaloupka, Acta Phys. Pol. A 121, 1026 (2012).

[31] A.M. Oleś, P. Horsch, L.F. Feiner, G. Khaliullin, Phys. Rev. Lett. 96, 147205 (2006).

[32] Y.-Q. Li, M. Ma, D.-N. Shi, F.-C. Zhang, Phys. Rev. B 60, 12781 (1999).

[33] W. Brzezicki, J. Dziarmaga, A.M. Oleś, Phys. Rev. Lett. 112, 117204 (2014).

[34] B. Kumar, Phys. Rev. B 87, 195105 (2013).

[35] W.-L. You, A.M. Oleś, P. Horsch, Phys. Rev. B 86 094412 (2012).

[36] W. Brzezicki, J. Dziarmaga, A.M. Oleś, Phys. Rev. Lett. 109, 237201 (2012).
[37] W. Brzezicki, J. Dziarmaga, A.M. Oleś, Phys. Rev. B 87, 064407 (2013); Acta Phys. Pol. A 126, A-40 (2014); W. Brzezicki, A.M. Oleś, Phys. Rev. B 83, 214408 (2011).

[38] J. Fujioka, T. Yasue, S. Miyasaka, Y. Yamasaki, T. Arima, H. Sagayama, T. Inami, K. Ishii, Y. Tokura, Phys. Rev. B 82, 144425 (2010).

[39] G. Khaliullin, P. Horsch, A.M. Oleś, Phys. Rev. Lett. 86, 3879 (2001); Phys. Rev. B 70, 195103 (2004).

[40] P. Horsch, A.M. Oleś, L.F. Feiner, G. Khaliullin, Phys. Rev. Lett. 100, 167205 (2008).

[41] C. Ulrich, G. Khaliullin, J. Sirker, M. Reehuis, M. Ohl, S. Miyasaka, Y. Tokura, B. Keimer, Phys Rev. Lett. 91, 257202 (2003); P. Horsch, A.M. Oleś, G. Khaliullin, Phys. Rev. Lett. 91, 257202 (2003).

[42] J. Sirker, A. Herzog, A.M. Oleś, P. Horsch, Phys. Rev. Lett. 101, 157204 (2008).

[43] K. Wohlfeld, M. Daghofer, A.M. Oleś, Europhys. Lett. 96, 27001 (2011).

[44] P. Bogdanski, M. Halaoui, A.M. Oleś, R. Frésard Phys. Rev. B 82, 195125 (2010).

[45] A. Nicholson, W. Ge, X. Zhang, J. Riera, M. Daghofer, A.M. Oleś, G.B. Martins, A. Moreo, E. Dagotto, Phys. Rev. Lett. 106, 217002 (2011).

[46] A.J.W. Reitsma, L.F. Feiner, A.M. Oleś, New J. Phys. 7, 121 (2005).

[47] G. Baskaran, S. Mandal, R. Shankar, Phys. Rev Lett. 98, 247201 (2007).

[48] S.K. Choi, R. Coldea, A.N. Kolmogorov, T. Lancaster, I.I. Mazin, S.J. Blundell, P.G. Radaelli, Y. Singh, P. Gegenwart, K.R. Choi, S.-W. Cheong, P.J. Baker, C. Stock, J. Taylor, Phys. Rev. Lett. 108, 127204 (2012).

[49] R. Comin, G. Levy, B. Ludbrook, Z.-H. Zhu, C.N. Veenstra, J.A. Rosen, Y. Singh, P. Gegenwart, D. Stricker, J.N. Hancock, D. van der Marel, I.S. Elfimov, A. Damascelli, Phys. Rev. Lett. 109, 266406 (2012).

[50] J. Chaloupka, G. Jackeli, G. Khaliullin, Phys. Rev. Lett. 105, 027204 (2010); Phys. Rev. Lett. 110, 097204 (2013).

[51] I. Kimchi, Yi-Z. You, Phys. Rev. B 84, 180407 (2011).

[52] T.A. Kaplan, P. Horsch, W. von der Linden, J. Phys Soc. Jpn. 58, 3894 (1989).

[53] F. Trousselet, P. Horsch, A.M. Oleś, W.-L. You, Phys. Rev. B 90, 024404 (2014).

[54] E. Sela, H.-C. Jiang, M.H. Gerlach, S. Trebst, Phys Rev. B 90, 035113 (2014).

[55] A.F. Albuquerque, D. Schwandt, B. Hetényi, S. Capponi, M. Mambrini, A.M. Läuchli, Phys. Rev. B 84, 024406 (2011).

[56] D. Gotfryd, A.M. Oleś, Acta Phys. Pol. A 127, 318 (2015).

[57] F. Trousselet, M. Berciu, A.M. Oleś, P. Horsch, Phys Rev. Lett. 111, 037205 (2013).

[58] W. Brzezicki, A.M. Oleś, M. Cuoco, arXiv:1408.1838; Phys. Rev. X 5, in press (2015). 